\title{
OPINIÓN INVITADA
}

\author{
La cumbre Kim-Trump y el acercamiento \\ entre las dos Coreas: Límites y expectativas
}

\section{The Kim-Trump Summit and the rapprochement between the two Koreas: Limits and expectations}

DOI: $10.32870 / m y c p . v 7 i 21.598$

Ulises Granados ${ }^{1}$

\section{Introducción}

El 12 de junio de 2018 el presidente de Estados Unidos, Donald Trump, y el presidente de la Comisión de Asuntos Estatales de la República Popular Democrática de Corea (Corea del Norte), Kim Jong-un, sostuvieron un encuentro en la isla de Sentosa en Singapur, la primera reunión, en la historia, entre los líderes en funciones de estos dos países. Esta reunión, histórica sin duda, produjo un comunicado conjunto de cuatro puntos: primero, el compromiso por establecer una nueva relación pacífica y próspera entre Estados Unidos y Corea del Norte; segundo, el compromiso para construir un régimen de paz duradero y estable en la Península Coreana; tercero, el propio compromiso del régimen de Pyongyang de trabajar hacia la completa desnuclearización de la península; y cuarto, la identificación y repatriación inmediata de los restos de prisioneros de guerra caídos en la Guerra de Corea (1950-1953) (Singapore Summit, 2018). Sobre estos puntos, las dos partes se han comprometido a implementarlos, y de lograrse, podría potencialmente terminarse con décadas de inseguridad regional resultado de este conflicto y uno de los pocos remanentes de la Guerra Fría.

La cumbre de Singapur, a su vez, ocurre mes y medio después de una particularmente importante reunión en la parte surcoreana de la zona desmilitarizada de Panmunjeom el 27 de abril entre el presidente surcoreano Moon Jae-in y el líder norcoreano. La Declaración de Panmunjeom, resultado de esta

1. Instituto Tecnológico Autónomo de México (ITAM), Programa de Estudios Asia Pacífico. Río Hondo 1, Col. Progreso Tizapán, Delegación Álvaro Obregón, C. P. 01080, Cuidad de México, México. ORciD http://orcid.org/0000-0002-7523-3592 Correo electrónico: ulises.granados@itam.mx 
voluntad política de los propios coreanos, marca la hoja de ruta, primero, hacia la posible co-prosperidad económica y la unificación intercoreana; segundo, hacia el fin de la tensión militar y del peligro de guerra a través, entre otras medidas, de la desnuclearización de toda la península; y tercero, hacia la creación de un régimen de paz permanente y sólido. Este acuerdo incluso prevé la participación de otros países involucrados en la Guerra de Corea, principalmente Estados Unidos y China, a favor de un posible tratado de paz.

Las dos cumbres representan un giro radical de años de amenazas de guerra y una escalada nuclear que mantiene a la comunidad internacional en permanente estado de tensión, y es por ello que ambas reuniones tienen un valor intrínseco. Estos encuentros no podrían entenderse si los descontextualizamos de la cada vez más peligrosa tensión derivada del hecho de que el régimen de Pyongyang ya posee, posiblemente, varias docenas de armas nucleares y algunas de hidrógeno (varias veces más potentes) y de una tecnología de misiles al parecer capaz de llegar a territorio continental estadounidense. Esta creciente tensión coincide también con la reciente llegada al poder de Donald Trump, quien, en un alarde de capacidad negociadora, primero amenazó con "hacerse cargo del problema norcoreano" y recientemente de sentarse a la mesa con Kim Jong-un para lograr la desnuclearización irreversible y verificable de la Península Coreana (léase, de Corea del Norte). Ahora el mandatario estadounidense repite a aliados y detractores por igual que ha resuelto el problema norcoreano.

Las preguntas necesarias, sin embargo, son: ¿estos acuerdos son reversibles o serán respetados? ¿Se puede alcanzar la paz y seguridad en la Península Coreana o estos acuerdos son más de forma que de sustancia? ¿Cómo se implementarán ante el usual déficit de confiabilidad existente entre todas las partes? ¿Qué papel desempeñan Japón, Rusia y China, aparentes observadores que históricamente han estado más involucrados de lo que ahora pareciera? Las respuestas, por un lado, dependerán enteramente de la voluntad personal de los líderes y de la honestidad de ellos a favor de la paz y seguridad; por otro lado, se puede dilucidar desde el tortuoso camino que se ha recorrido en la península, en particular de los programas bélicos norcoreanos, de un análisis de la geopolítica global y regional y sus implicaciones en el noreste de Asia, y del éxito o fracaso de los sucesivos proyectos de contención contra las actividades nucleares del régimen norcoreano, principalmente de las sanciones del Consejo de Seguridad de la Organización de las Naciones Unidas. 


\section{Avance de los programas norcoreanos}

Si bien Pyongyang cuenta con programas de armamento nuclear, biológico, químico y de misiles, el nuclear es actualmente el más preocupante por ser el más avanzado, ser muy propenso a la proliferación internacional, y por ser consecuencia natural de un programa pacífico para generación de energía. Iniciado incluso antes del armisticio de 1953 y desarrollado en diferentes fases en la década de los años sesenta, el programa nuclear avanzó con apoyo soviético y sin el apoyo inicial de China (Broad, Sanger \& Bonner, 2004), aunque en las siguientes dos décadas avanzó con investigación y tecnología virtualmente propia, incluyendo la construcción del reactor de Yongbyong e instalaciones de procesamiento de uranio en los años ochenta. Bajo el argumento de utilizar la energía nuclear con fines pacíficos, Corea del Norte se adhirió al Tratado de No Proliferación Nuclear (TNP) desde 1985 y aceptó el sistema de salvaguardas del Organismo Internacional de Energía Atómica (OIEA) en 1992. Sin embargo, tan sólo un año después se descubrió que había reprocesado plutonio en 1989, 1990 y 1991 y ante la petición de inspecciones del OIEA para visitar presuntos sitios de reprocesamiento, Corea del Norte declaró varias instalaciones nucleares como militares y amenazó con su retiro del TNP. Es importante recordar que en la crisis de 1994 entre la administración Clinton y Kim Il Sung — que eventualmente desembocó en un acuerdo-, el ex presidente Jimmy Carter, de visita en Pyongyang, desempeñó un papel activo para que Norcorea aceptara en ese entonces la implementación de la "Declaración Conjunta Norte Sur sobre la Desnuclearización de la Península Coreana" de 1992. Este esfuerzo diplomático de alto nivel impulsó un proceso que, sin embargo, fracasó nueve años después.

Aquí la proliferación nuclear en el mercado negro parece haber beneficiado particularmente a Corea del Norte. Desde que en 2002 se descubrió que el régimen de Pyongyang podría poseer un programa ilícito de uranio altamente enriquecido, emergió información pocos años después sobre las actividades del científico nuclear paquistaní Abdul Qadeer Khan de que habría transferido tecnología nuclear a Norcorea (al igual que a Irán y Libia) a cambio de recibir tecnología de misiles para su nación (Broad, Sanger \& Bonner, 2004). Y desde luego la importancia del programa de misiles de Corea del Norte, ya que un programa de armamento nuclear está incompleto sin un programa que proporcione los vehículos de reingreso (misiles balísticos) de las cargas nucleares. Así, durante las décadas de los años ochenta y noventa avanzó 
un ambicioso programa de misiles bajo el liderazgo de Kim Il Sung con 15 lanzamientos de 1984 a 1993. Varios años después, bajo el liderazgo de Kim Jong Il, Corea del Norte lanzó en 1998 un misil Taepodong I de alcance medio sobre espacio aéreo japonés (Gittings, 1998). Desde entonces, la preocupación por el ritmo del avance en estos dos programas es particularmente preocupante ya que coinciden las pruebas nucleares subterráneas —la primera en 2006con pruebas de versiones mejoradas de misiles Taepodong 2, Nodong, Unha 2, Unha 3, Pukkuksong 1, Pukkuksong 2, y Hwasong (Musudan).

Desde el año 2006 Corea del Norte ha realizado seis pruebas nucleares: en 2006 (con una carga de menos de un kilotón), 2009 (5.4 kilotones), 2013 (16 kilotones), dos en 2016 (17 y 25 kilotones, respectivamente) y 2017 (hay estimaciones de hasta 280 kilotones). Y el momento de decidir sobre estas pruebas subterráneas ha sido usado, a su vez, como el último instrumento de disuasión, provocación o represalia. La primera prueba nuclear de 2006 y el lanzamiento previo de misiles fueron ordenados a pocos meses de que el Departamento del Tesoro estadounidense y el Gobierno de Macao congelaran unas 50 cuentas norcoreanas en el Banco Delta Asia de Macao. Asimismo, la detonación de 2009 puede ser interpretada como una medida de presión ante el aumento en las sanciones de la Organización de las Naciones Unidas (ONU) y de Estados Unidos derivadas del impasse en el protocolo de verificación del programa nuclear norcoreano que se creyó irreversible tras el desmantelamiento de las instalaciones de Yongbyon desde 2008 en el marco de los acuerdos de seis países. Esta vez la decisión de la administración Bush de eliminar a Corea del Norte de la lista de países que apoyan el terrorismo no impidió el avance en los programas nuclear y de misiles.

La prueba nuclear de 2013 (el 12 de febrero) fue también cuidadosamente programada para el final de la administración del presidente surcoreano Lee Myung-bak (2008-2013), conocido por su línea dura contra Norcorea y diametralmente opuesto a la Sunshine Policy de Kim Dae-jung (1998-2003), al acercamiento emprendido hacia Pyongyang por su sucesor Roh Moo-hyun (2003-2008), y en clara advertencia a la entrante presidenta Park Gyeun-hee (2013-2017). Más aún: las pruebas nucleares y de misiles desde 2012 -las primeras bajo el pretexto de lanzar satélites de comunicación- envían el claro mensaje de que el nuevo régimen de Kim Jong-un, desde la muerte de su

2. Mil toneladas de TNT. En comparación, la bomba detonada en Hiroshima tuvo una carga de 15 kilotones. 
padre Kim Jong Il en 2011, "busca estas formas asimétricas de garantizar su propia seguridad" (Gwertzman, 2013). Así, 89 lanzamientos de misiles y tres pruebas nucleares se han registrado de 2011 a 2017 a fin de perfeccionar la tecnología utilizada y dejar claro que el actual líder norcoreano es todo menos débil e inexperto, como aseguraban evaluaciones iniciales de su régimen. La prueba nuclear de enero de 2016, aclamada por el régimen de Pyongyang como una bomba de hidrógeno (pero que probablemente fue un dispositivo menor de fisión), parece haber sido la respuesta a la petición de Estados Unidos de incluir la desnuclearización norcoreana como parte del paquete de discusiones para la firma de un eventual tratado de paz, este último solicitado poco antes por la parte norcoreana (Reuters, 2016). Varios meses después se ordena la segunda prueba del año, y a pesar de cinco resoluciones de sanciones del Consejo de Seguridad —las más recientes con la venia incluso de China-, esta explosión reveló la posibilidad de que el régimen norcoreano ya puede producir a voluntad, como lo afirman ellos mismos, una gran cantidad de ojivas nucleares miniaturizadas de alto poder destructivo. Asimismo, fue un claro mensaje a la próxima administración estadounidense antes de las elecciones presidenciales sobre la determinación de obligarlos a negociar un acuerdo de paz ante el fait accompli de una poderosa potencia nuclear.

Desde finales de 2017 se ha formulado la pregunta sobre la capacidad de Corea del Norte de negociar, directamente con Estados Unidos, desde una postura de particular fuerza por los dramáticos avances en sus programas de misiles y nuclear. Luego de repetidos lanzamientos fallidos en 2016 y 2017, Corea del Norte pudo finalmente probar un nuevo motor para misil ICMB en junio de 2017, su primer misil intercontinental Hwasong 14 en julio, y otro en noviembre del mismo año con un rango potencial de 13 mil kilómetros, es decir, con alcance a todo Estados Unidos continental. Pero el momento decisivo en el programa norcoreano se reveló en septiembre cuando detonó su primer bomba de hidrógeno, ya que no sólo elevó el nivel de protestas de la comunidad internacional y del Consejo de Seguridad ante la real amenaza global que el régimen representa, sino que además con esa explosión parece haber dejado inutilizado el sitio de pruebas norcoreano de Punggye-Ri cerca de la frontera con China. Esta situación es muy relevante para todo el mundo: Pyongyang ha ofrecido como una gran concesión (el atestiguar la destrucción de túneles subterráneos de pruebas nucleares por parte de la prensa internacional, mas no así de inspectores expertos) algo que en realidad podría representar un chantaje velado a Estados Unidos, a su vecino China 
y al mundo ante la posibilidad de que otra explosión libere radiación a la atmósfera con consecuencias catastróficas. La abrumadora realidad es que Corea del Norte mantiene un arsenal no declarado de armamento nuclear que incluye bombas de hidrógeno y misiles operacionales para alcanzar a su enemigo, lo que representa una posición muy cómoda que le permitió desde inicios de 2018 en el contexto de las Olimpiadas de Invierno en Pyeongchang un enorme margen de maniobra ante los jóvenes gobiernos de Moon Jae-in y de Donald Trump. En su discurso televisado el 29 de noviembre de 2017, un día después del lanzamiento exitoso del misil intercontinental, Kim Jong-un declaró: "la totalidad de la parte continental (de Estados Unidos) está dentro del rango de nuestro ataque nuclear y el botón nuclear está en mi escritorio todo el tiempo" (New Year's, 2018).

\section{¿Cómo contener a Corea del Norte?}

Los programas de destrucción masiva tienen un problema particularmente difícil de encarar: los descubrimientos científico-tecnológicos para fisionar materiales, aislar isótopos y cultivar patógenos o productos químicos tóxicos son irreversibles, no se pueden "des-aprender" y lo más sensato es mantener a buen resguardo este conocimiento. En consecuencia, este avance presenta al mundo los problemas de la proliferación y la posibilidad de que cualquier nación se convierta en potencia nuclear. Desde la década de los cincuenta Corea del Norte ha desarrollado tecnología nuclear con fines pacíficos, pero las enseñanzas de la disuasión nuclear, que desde los setenta condicionó la mentalidad de supervivencia de países como India y Pakistán, fue aceptada por el régimen de Pyongyang y considerada como su mejor opción de supervivencia desde el fin de la Guerra Fría.

El primer intento de la comunidad internacional de contener los programas nuclear y de misiles de Corea del Norte, y al mismo tiempo de garantizar el inalienable derecho del uso de energía nuclear para fines pacíficos (como lo garantiza el propio TNP) tuvo lugar en 1994-1995, cuando Estados Unidos, Corea del Sur y Japón llegaron a un acuerdo con Corea del Norte para sustituir los reactores de grafito tipo Magnox por dos reactores de agua ligera desde 2003 y a cambio de un ambicioso proyecto de asistencia energética y económica mediante un consorcio llamado Organización para el Desarrollo Energético de la Península (KEDO, por sus siglas en inglés). El Acuerdo Marco, resultado de estas negociaciones, pudo contener temporalmente la producción 
de plutonio y poner los reactores existentes bajo el sistema de salvaguardas del oIEA (The U. S.-North Korean, 2017). Sin embargo, el lanzamiento del misil Taepodong en 1998 y la recomendación dentro de la administración Clinton de forzar a Pyongyang a un nuevo acuerdo derivó en la continuación de los programas nuclear y de misiles norcoreanos, en particular desde que el recién electo presidente George Bush incluyó en 2002 a Corea del Norte como parte del "eje del mal" a un año de los ataques terroristas del 9/11. Hasta 2005 más de 10 naciones invirtieron un total de más de dos mil 500 millones de dólares en el consorcio.

Un segundo esfuerzo fue encabezado por Corea del Sur con la puesta en marcha de la Sunshine Policy del presidente Kim Dae-jung en 1998 y continuada por su sucesor Roh Moo-hyun de 2003 a 2008, una política de involucrar al norte en mecanismos de cooperación y desarrollo. Esta política de acercamiento resultó en dos cumbres intercoreanas en Pyongyang, la primera entre Kim Jong-il y Kim Dae-jung en junio de 2000 (que le valió a Kim Dae-jung el premio Nobel de la Paz), y la segunda entre Kim Jong-il y Roh Moo-hyun en octubre de 2007. Estas cumbres intentaron avanzar en el proceso de reunificación, de una península libre de armas nucleares, y en un pacto de paz permanente. Además, se fomentaron empresas conjuntas (como el complejo industrial Kaesong), turismo surcoreano en el norte (en particular en el monte Kumgang) y esporádicas reuniones de familias separadas por la guerra. Sin embargo, el escándalo que representó saber que Corea del Sur, a través de la empresa Hyundai, pagó 500 millones de dólares al líder norcoreano en 2000 (AFP \& JIJI, 2018), y el alto escepticismo en la comunidad internacional a un año de la primera prueba nuclear norcoreana en 2006, eclipsaron esta política. A la llegada al poder de Lee Myung-bak en 2008 la Sunshine Policy había llegado a su fin.

El tercer intento, más coordinado, nació a partir de la denuncia estadounidense de que Pyongyang violó el Acuerdo Marco de 1994 y del anuncio de Corea del Norte de retirarse del Tratado de No Proliferación Nuclear en 2003 ante informes de que continuaba con su programa de enriquecimiento de uranio, lo cual llevó a diversos países a involucrar directamente y de forma multilateral al régimen de Pyongyang en las llamadas Conversaciones a Seis Bandas (Six Party Talks) (Council on Foreign Relations, 2013). Este foro multilateral, con la participación de las dos Coreas, Estados Unidos, China, Rusia y Japón, tuvo su vida útil de 2003 a 2009, y aunque formalmente no ha terminado sus funciones y llegó a producir en septiembre de 2005 un 
acuerdo en el que Corea del Norte aceptaría abandonar su programa nuclear, se encuentra virtualmente suspendido tras seis rondas de negociaciones por la falta de acuerdo en los mecanismos de verificación de desmantelamiento de instalaciones nucleares, y principalmente por la confirmación de cinco de las seis partes de que Corea del Norte no tenía la intención real de desnuclearizarse. El régimen de Pyongyang, por su parte, lanzó en abril de 2009 un misil Taepodong 2, realizó su segunda prueba subterránea un mes después, y anunció en 2010 de la existencia de una instalación para el enriquecimiento de uranio, mientras que intermitentemente ha ofrecido la suspensión de sus pruebas nucleares y el acceso de monitores del OIEA a cambio de ayuda alimentaria de Estados Unidos.

Desde la primera prueba nuclear norcoreana en 2006, la opción más eficaz de contención, si bien de efecto lento pero inexorable, es la serie de sanciones aprobadas mediante resoluciones del Consejo de Seguridad de la ONU. El contenido central de las principales sanciones contra Corea del Norte es el siguiente:

- Números 1695 y 1718 (de julio y octubre de 2006), la primera prohibiendo compraventa de material y tecnología para misiles, y la segunda imponiendo un primer embargo económico y comercial —incluyendo bienes de lujo - en castigo por la primera prueba nuclear.

- Número 1874 (junio de 2009), extiende las sanciones al ámbito financiero y autoriza inspecciones de carga a buques norcoreanos en represalia por la segunda prueba nuclear.

- Número 2087 (enero de 2013), condena el lanzamiento norcoreano de misil en diciembre de 2012.

- Número 2094 (marzo de 2013), condena la tercera prueba nuclear e impone sanciones financieras a varias empresas y prohibición de viaje a personas vinculadas con el sector militar.

- Número 2270 (marzo de 2016), en respuesta a la cuarta prueba nuclear, ordena inspecciones a buques norcoreanos, un embargo total de armas, mayores restricciones de importaciones a Corea del Norte y la expulsión de varios diplomáticos norcoreanos.

- Número 2321 (septiembre de 2016), en respuesta a la quinta prueba nuclear, limita exportaciones norcoreanas de carbón y diversos productos naturales, amplía el embargo de armas y la vigilancia marítima, e impone restricciones de personal diplomático en el exterior, entre otras. 
- Número 2356 (junio de 2017), identifica a personas y empresas sujetas a sanciones particulares.

- Número 2371 (agosto de 2017), en respuesta al lanzamiento de misiles en 2017, en particular el ICBM de julio, ordena nuevas restricciones a exportaciones de carbón, materias primas y alimentos marinos, así como prohibición de contratar nuevos trabajadores norcoreanos.

- Número 2375 (septiembre de 2017), en respuesta a la sexta prueba nuclear, ordena una reducción drástica de importaciones norcoreanas de petróleo, prohíbe la venta de sus textiles e impone mayores restricciones a trabajadores norcoreanos en ultramar.

- Número 2397 (diciembre de 2017), en respuesta al lanzamiento del misil Hwasong 15, recrudece las sanciones de la resolución 2375, algo calificado por Corea del Norte como un "acto de guerra".

Como es patente, las sanciones son cada vez más severas y son respuesta directa a los lanzamientos de misiles, al presunto lanzamiento de satélites (que en realidad han sido intentos de probar misiles con potenciales cargas militares), al retiro de las Conversaciones a Seis Bandas en 2009, pero sobre todo a las detonaciones nucleares subterráneas realizadas en 2006, 2009, 2013, en dos ocasiones en 2016, y en 2017. La prueba nuclear de septiembre de 2017, en particular, ha tenido como respuesta no sólo la resolución del Consejo de Seguridad, sino el recrudecimiento de sanciones unilaterales por parte de Estados Unidos, la Unión Europea y Japón, e incluso de la expulsión de altos diplomáticos norcoreanos de varios países incluyendo México, Kuwait, Perú y España.

Las sanciones de Estados Unidos han sido particularmente severas desde 2008, ya que están diseñadas para cortar las fuentes de financiamiento y de combustibles a Corea del Norte, e incluyen desde 2018 a entidades y empresarios de China, Hong Kong, Taiwán, Panamá y Singapur (BBC News, 2018). La Unión Europea actualmente mantiene sanciones contra 58 personas y 10 empresas norcoreanas (North Korea: EU, 2018), en tanto que Japón mantenía hasta finales de 2017 un total de 62 personas y 56 grupos comerciales norcoreanos bajo sanciones unilaterales (Yamaguchi, 2017). La ejecución de sanciones, dentro y fuera del marco de la ONU, es vital para forzar al régimen de Pyongyang a negociar, y parecen haber surtido efecto ante la desesperación de allegarse fondos y combustible (desde finales de 2017 varias fuentes de inteligencia reportan avistamientos de barcos norcoreanos 
y cisternas de otros países - ¿chinos y rusos? - transfiriendo productos y combustible en alta mar). ${ }^{3}$

\section{La dimensión geopolítica regional y global}

A pesar de que parece que la negociación para una paz duradera y una Península Coreana desnuclearizada es entre Estados Unidos y Corea del Norte, el problema es tanto regional como global, y una solución real no puede soslayar los intereses y objetivos de Corea del Sur, Japón, Rusia y China. De progresar el acuerdo Trump-Kim y los objetivos trazados en la reciente cumbre intercoreana, los intereses de estos países moldearán el avance, su calendario o su eventual fracaso. Evidentemente el tema de la desnuclearización y el combatir la proliferación en su acepción más amplia (incluyendo tecnología de misiles y armamento biológico) desde Norcorea han sido prioridades para Washington, mas no así para todas las partes involucradas.

Corea del Sur es el país más vulnerable a la inestabilidad en la península; consciente de que Corea del Norte no necesitaría de armamento nuclear para traspasar la zona desmilitarizada e invadir al vecino del sur, Seúl ha utilizado un enorme capital político y financiero para inducir un diálogo con Pyongyang desde 1998. Sin embargo, la presencia de fuerzas militares estadounidenses es el garante de la supervivencia del Estado surcoreano en un escenario de guerra, y por lo mismo, a pesar de que formalmente Corea del Sur no tiene armamento nuclear estadounidense desde 1991, es improbable que acepte el retiro de las bases o los ejercicios conjuntos con Washington (suspendidos recientemente por el presidente Trump) o el sistema THAAD de protección contra misiles. Por otro lado, debido a la herencia común con la parte norcoreana, es posible que Seúl continúe apoyando algún tipo de unificación y promoviendo el desarrollo económico del norte para paliar el costo de una posible unión.

Japón también se siente especialmente vulnerable a los programas nuclear, biológico y de misiles de Corea del Norte, por lo que ha patrocinado diversas resoluciones de sanción contra el régimen de Pyongyang en el Consejo de Seguridad (en 1998, en 2009 y en varias ocasiones, en 2017, misiles norcoreanos han surcado el espacio aéreo japonés). Asimismo, y en

3. Entre las diversas fuentes están: Serene Cheong \& Dan Murtaugh, 2018, North Korea keeps sneaking in oil with secret ship transfers. Stars and Stripes, 4 de enero. Disponible en https://www. stripes.com/news/pacific/north-korea-keeps-sneaking-in-oil-with-secret-ship-transfers-1.505201 
el marco de la histórica hostilidad entre los dos países, Japón mantiene su intención de resolver los casos pendientes de nacionales secuestrados en el pasado por agentes norcoreanos. Así, Tokio se congratula de la tercera cumbre intercoreana y de la cumbre de Singapur ya que garantizan por el momento la suspensión de lanzamientos de misiles, pero se mantiene muy cauto ante la probada actitud engañosa norcoreana sobre temas pendientes bilaterales. Cualquier proceso de normalización (iniciado mediante la Declaración de Pyongyang de 2002) debe involucrar, desde la perspectiva japonesa, una solución a los casos pendientes de secuestro en décadas pasadas (Ministry of Foreign Affairs Japan, 2017, p. 33), tanto como la repatriación de los restos de soldados estadounidenses.

Tanto Rusia como China, vecinos del régimen norcoreano, consideran que sus acciones en el conflicto de la península deben responder a sus respectivos intereses de moldear el ambiente de seguridad en el este de Asia y de garantizar una posición de autoridad en la escena internacional en general, y por lo mismo, más allá de pronunciarse a favor de la paz y estabilidad en el noreste de Asia, su política para la península es sólo parte de su estrategia de presencia global. Moscú está consciente de que la existencia del régimen norcoreano es vital para la seguridad de China, por lo que no hay enfrentamientos entre Beijing y Moscú sobre las sanciones hacia Corea del Norte. A cambio, Rusia espera el "reconocimiento de los intereses rusos en áreas de gran preocupación para Moscú, como el Medio Oriente” (Lukin, 2017). El interés de Rusia hacia la desnuclearización de Corea del Norte y un acuerdo de paz permanente le retribuiría no sólo la garantía de estabilidad estratégica de sus propios arsenales emplazados en el este de Asia (ya que se podría frenar una carrera armamentista nuclear) sino en la posibilidad de comercio, inversión e infraestructura para conectar la península con sus regiones del extremo oriente. El mismo hecho de que no tenga tanta influencia económica sobre Pyongyang hace de Moscú un "intermediario honesto" sin aparentes grandes pretensiones. La visita del canciller ruso Sergei Lavrov a Pyongyang para ayudar a salvar la cumbre de Singapur del 12 de junio de 2018, y la aceptación de Kim Jong-un de una visita a Moscú en el año (Stevenson, 2018) revelan esta capacidad diplomática rusa.

Para China, 12 años de pruebas nucleares han minado la relación con Pyongyang por la inestabilidad que han causado; sin embargo, Beijing sabe que una frontera común con una Corea del Norte que pudiera colapsar por una guerra o un cambio de régimen violento sería desastroso en términos 
humanitarios y de migraciones masivas. El gobierno chino apuesta a la paz y estabilidad en el noreste de Asia para la implementación exitosa de su propia estrategia de desarrollo. Las implicaciones del aumento de la tensión en la península ya se han dejado ver: Beijing considera que el emplazamiento de radares en el sistema antimisiles THAAD en Corea del Sur puede alterar el balance estratégico de la fuerza convencional y nuclear china en la región, más que detener las trayectorias de los misiles norcoreanos - la decisión de Corea del Sur de albergar el sistema condujo en 2016 a un severo deterioro en las relaciones Beijing-Seúl (Panda, 2017).

Para Corea del Norte, China sigue siendo su principal aliado (Kim Jong-un se reunió tres veces entre marzo y junio de 2018 con el presidente Xi Jinping), y Estados Unidos sabe que no puede ignorar los intereses de China, en la región y a escala global (principalmente comercio y seguridad internacional), al momento de negociar los términos de la desnuclearización de la península. De hecho, China tiene un margen de acción más grande del que se piensa: puede ayudar a cambiar el sistema económico norcoreano con reformas similares a las suyas, puede ser el garante de la seguridad del arsenal nuclear norcoreano, puede ofrecer ayuda energética y negociar el levantamiento parcial de las sanciones, y puede negarse o aceptar ser firmante de un tratado de paz que termine con la misma Guerra de Corea.

\section{La cumbre Trump-Kim: ¿la solución ideal?}

A pesar de las grandes expectativas de un cambio para bien en la situación de la Península Coreana, la cumbre de Singapur fue el resultado de una diplomacia apresurada y arriesgada por parte de Estados Unidos y Corea del Norte, lo cual podría minar en esencia el impacto a largo plazo. Ante esto, destaca el papel protagónico que todavía ejerce Corea del Sur, mediante la decisión del presidente Moon Jae-in de convencer a Corea del Norte de reiniciar el diálogo intercoreano desde principios de 2018 en respuesta de la visita de la hermana de Kim Jong-un, Kim Yo-jong, a la inauguración de los Juegos Olímpicos de Invierno en febrero. Esta notable distensión revela que la estabilidad de la península es un imperativo de los propios coreanos y sobre el interés de que ambos gobiernos, del norte y del sur, preserven la centralidad en el proceso. En este evento deportivo, sin embargo, se vio una actitud reservada y evasiva por parte del vicepresidente estadounidense, Mike Pence, ante la delegación norcoreana, lo que dejó evidente que Estados Unidos no estaba preparado 
aún para sentarse a negociar. Y aun así, tras la justa olímpica, el gobierno surcoreano emprendió una frenética cruzada diplomática para organizar la cumbre intercoreana y mediar en un encuentro Trump-Kim, enviando una delegación a Corea del Norte. Mientras tanto, Kim Jong-un, al tiempo que aceptó participar en la cumbre, inició un rápido acercamiento con China desde el primero de sus tres viajes a China en marzo, en un claro despliegue de habilidades diplomáticas con su principal aliado.

Por su parte, Donald Trump en sólo medio año ha decidido cambiar su temperamental retórica de insultos contra Kim Jong-un (en Twitter) desde finales de 2017, a llamarlo un negociador muy valioso e inteligente durante la cumbre. En estos seis meses, en preparación de la propia cumbre (así como de la inminente decisión de retirarse del acuerdo nuclear con Irán y de las sanciones comerciales contra aliados y adversarios por igual, entre otras razones), el presidente Trump sustituyó al secretario de Estado Rex Tillerson por el ex jefe de la CIA, Mike Pompeo, así como al general H. R. McMaster por el embajador John Bolton como asesor de Seguridad Nacional. La decisión de nombrar a Bolton, en particular, afectó brevemente los planes de la cumbre de Singapur, pero sí reveló claramente la lógica del proceso de desnuclearización como es percibido por Estados Unidos. En entrevista ofrecida en abril de 2018, Bolton se refirió al modelo libio de 2003-2004 de negociaciones de desnuclearización como una opción para el caso norcoreano, lo cual fue interpretado inmediatamente por el régimen de Pyongyang como un modelo de "primero desnuclearizarlos, luego destruirlos". La referencia del modelo libio fue repetida posteriormente por el vicepresidente Pence en mayo, desencadenando insultos del equipo negociador norcoreano y a su vez provocando el anuncio de Trump de la cancelación de la cumbre. Este episodio, a pesar de haber sido minimizado rápido por la Casa Blanca, refleja tanto la fragilidad de la diplomacia intramuros y el carácter incendiario de los líderes, como la real intención de Estados Unidos de ver pasos concretos en la desnuclearización de Corea del Norte antes del levantamiento de sanciones unilaterales y multilaterales.

Por si esto no fuera por demás complicado, la administración Trump enfrenta el reto de continuar con su política comercial hostil hacia sus propios aliados al tiempo que intenta garantizarles, en particular a Japón y Corea del Sur, que protegería sus intereses en las negociaciones frente al régimen de Pyongyang. Tokio está particularmente interesado en que el tema de la repatriación de los restos de soldados estadounidenses también incluya la 
solución de los casos de japoneses secuestrados por Norcorea en las décadas pasadas, mientras que Seúl ve con cautela el reciente anuncio de la suspensión de cualquier ejercicio militar conjunto con Estados Unidos como lo declaró Trump en junio.

\section{El camino por delante}

Indudablemente la diplomacia es el único camino alternativo a la confrontación militar en la Península Coreana, y los acuerdos convenidos en la cumbre de Singapur, por vagos que sean, representan un antídoto al acelerado programa nuclear norcoreano que ya ha llegado a un punto sin retorno. A pesar de que no se puede descartar que el espíritu de diálogo está cimbrado en las dos personalidades megalómanas de Trump y Kim con propias aspiraciones personales (hasta ahora se desconoce el contenido de la conversación privada entre estos dos líderes en la isla de Sentosa el día de la cumbre), la comunidad internacional puede estar segura, por ahora, de que la tensión bajará en los próximos meses. Ante todo esto, es importante no perder de vista que esta reunión, por histórica que sea, no es un fin o un documento legalmente vinculante - como un tratado de paz formal一, sino el inicio de un proceso perfectible.

Sin embargo, la apuesta es alta: un proceso de negociaciones iniciado y encabezado por los líderes y no al revés - como un proceso tradicional de negociaciones diplomáticas debe ser-, cierra la puerta a un mayor margen de concesiones. Ahora es más difícil una alternativa a este proceso, aunque Corea del Sur podrá obtener paralelamente beneficios más modestos, pero de mayor plazo, como una cooperación económica sólida al estilo de la Sunshine Policy de Kim Dae-jung.

Tampoco hay que perder de vista que, desde una perspectiva estratégica, para Corea del Norte es casi imposible renunciar a sus programas de armamentos nuclear, biológico, químico y de misiles, a cambio de la mera promesa de ayuda económica y energética a futuro y bajo las severas sanciones de la comunidad internacional. Simplemente ha invertido mucho capital político y económico para llegar hasta donde ha llegado, por lo que seguramente intentará obtener las mayores concesiones, de todos y cada uno de los países involucrados, antes de permitir la verificación de su desnuclearización. Frente a esto, Estados Unidos se decanta por lo contrario: desnuclearización total, permanente y verificable, antes del retiro de sanciones. Es sintomático que 
en la cumbre de Singapur, entre quienes acompañaron a Trump en la mesa estuvieran precisamente el ex titular de la cIA Mike Pompeo, el controversial halcón John Bolton y el general John Kelly como su jefe de Gabinete de la Casa Blanca.

Ante esto, el panorama no es tan alentador. Como se presentó líneas arriba, durante más de 25 años el régimen de Pyongyang se ha retirado consistentemente de los diversos mecanismos y procesos de cooperación destinados a desnuclearizar Corea del Norte y reincorporarlo en el concierto de naciones, y ha avanzado inexorablemente hacia la posesión de una fuerza disuasoria nuclear capaz de obligar a negociar a Estados Unidos. La historia de las negociaciones con el régimen de Pyongyang demuestra que Kim Jongun puede retirarse de los acuerdos de la cumbre de Singapur en cualquier momento, y que el temperamento de los dos líderes puede enterrar el acuerdo.

Indudablemente, el camino a seguir debe sobrepasar el mero compromiso de cumplir lo prometido en la cumbre de Singapur, y se debe continuar con las reuniones bilaterales en los próximos meses. Aceptar que esto es parte de un largo proceso ayuda a entender por qué Washington ha aceptado vagas promesas, por qué no presionó en el tema de derechos humanos en Norcorea, por qué elevó intencionalmente la imagen internacional de Kim Jong-un, e incluso ahora ofrece suspender ejercicios militares conjuntos con Corea del Sur. Frente a las críticas de que Trump concedió más de lo que obtuvo, habrá que reconocer a su administración cierto grado de visión estratégica (con o sin el "modelo libio" en mente) al negociar con Kim Jong-un.

Esta visión estratégica, sin embargo, implica que el proceso de eventual desnuclearización deba insertar a los países involucrados ofreciendo, en diversos grados, incentivos para presionar al régimen de Pyongyang. En primer lugar, se deben mantener las sanciones multilaterales, aunque algunas, las más severas que han casi sofocado totalmente los flujos energéticos, laborales y financieros, podrían ser atemperadas a corto plazo, dependiendo de los pasos iniciales que realice el liderazgo norcoreano. Más aún, como señalan Victor Cha y Katrin Fraser (2018), Estados Unidos debe fortalecer la actual coalición global no sólo para galvanizar la opinión del Consejo de Seguridad, sino para asegurar la no proliferación desde territorio norcoreano. Al mismo tiempo, se debe garantizar la fuerza de las alianzas militares con Japón y con Corea del Sur, así como el compromiso de Washington con Tokio de proteger los propios intereses japoneses relacionados con el programa de misiles, la proliferación y los casos de secuestro. Esta visión de coordinación de las políticas de Seúl, 
Tokio y Washington debe confirmar que la permanente crisis norcoreana ha sido parte de un delicado balance estratégico en el noreste de Asia con China y Rusia como contrapesos.

Por su parte, China y Rusia podrían involucrarse más abiertamente en el proceso de verificación con Corea del Norte, invocando un foro permanente similar a las Conversaciones a Seis Bandas auspiciadas por Beijing. A corto plazo, el cumplimiento de las sanciones por parte de Beijing y Moscú es crucial para lograr los objetivos de la cumbre de Singapur o posteriores documentos, y a largo plazo estas dos potencias podrán tener un papel protagónico en la eventual salvaguarda del arsenal nuclear norcoreano y del despegue económico de Norcorea que podría allanar el camino hacia una reunificación de algún tipo. Washington debe reconocer que para estos dos países la crisis norcoreana no sólo puede ser un eficaz instrumento para elevar sus respectivas capacidades de liderazgo global, sino que también tienen la capacidad de descarrilar el proceso, no sólo de desnuclearización, sino también de apoyo económico a Pyongyang, de un tratado de paz y de una posible reunificación de la península.

En resumen, tanto la tercera cumbre intercoreana como la cumbre de Singapur ofrecen la posibilidad de una nueva etapa de distensión en la Península Coreana, similar a la etapa de 1998 a 2007, aunque la ecuación ha sido inexorablemente modificada por el armamento nuclear norcoreano, por dos nuevos líderes intempestivos a los dos extremos del océano Pacífico y por nuevos intereses internacionales que moldean las políticas de los países involucrados. El camino se ha abierto. Dependerá de las políticas de diversos líderes en los próximos meses el que la región sea un escenario de "fuego y furia" o de "paz duradera". Ante todo, cautela y mucha paciencia.

\section{Referencias bibliográficas}

AfP, \& JIJI. (2018, 27 de abril). Sunshine Policy' and scandal features of previous inter-Korean summits. The Japan Times. Recuperado de https:// www.japantimes.co.jp/news/2018/04/27/asia-pacific/sunshine-policyscandal-features-previous-inter-korean-summits/\#.w0tzcdjkiim

$B B C$ News. (2018, 23 de febrero). Us to impose 'largest ever' N Korea sanctions. BBC News. Recuperado de https://www.bbc.com/news/world-uscanada-43173487

Broad, W. J., Sanger, D. E., \& Bonner, R. (2004, 12 de febrero). A Tale of Nuclear Proliferation: How Pakistani Built His Network. The New York Times. 
Recuperado de https://www.nytimes.com/2004/02/12/world/a-tale-ofnuclear-proliferation-how-pakistani-built-his-network.html

Cha, V., \& Fraser, K. K. (2018). The Right Way to Coerce North Korea. Foreign Affairs. Recuperdo de https://www.foreignaffairs.com/articles/northkorea/2018-04-01/right-way-coerce-north-korea

Council on Foreign Relations. (2013, septiembre). The Six Party Talks on North Korea's Nuclear Program. Council on Foreign Relations. Recuperado de https://www.cfr.org/backgrounder/six-party-talks-north-koreasnuclear-program

Gittings, J. (1998, 1 de septiembre). North Korea fires missile over Japan. Hardliners in Pyongyang 'overplay their hand'. The Guardian. Recuperado de https://www.theguardian.com/world/1998/sep/01/northkorea

Gwertzman, B. (2013, 31 de enero). North Korea's Nuclear Needs [Entrevista a Victor D. Cha]. Council on Foreign Relations. Recuperado de https://www. cfr.org/interview/north-koreas-nuclear-needs

Lukin, A. (2017, 29 de noviembre). Nuclear Weapons and Russian-North Korean Relations. Foreign Policy Research Institute. Recuperado de https:// www.fpri.org/article/2017/11/nuclear-weapons-russian-north-koreanrelations/

Ministry of Foreign Affairs Japan. (2017). Diplomatic Bluebook 2017. Tokio: MOFA.

New Year's Address. (2018, 1 de enero). North Korea Leadership Watch. Recuperado de http://www.nkleadershipwatch.org/2018/01/01/new-yearsaddress/

North Korea. (April, 2018, April). The Nuclear Threat Initiative. Recuperado de http://www.nti.org/learn/countries/north-korea/nuclear/

North Korea: EU sanctions 17 DPRK nationals involved in activities aimed at evading sanctions. (22 January 2018). Council of the European Union. Recuperado de http://www.consilium.europa.eu/en/press/press-releases/2018/01/22/north-korea-eu-sanctions-17-dprk-nationals-involvedin-activities-aimed-at-evading-sanctions/

Panda, A. (2017, 13 de noviembre). China and South Korea: Examining the Resolution of the THAAD Impasse. The Diplomat. Recuperado de https:// thediplomat.com/2017/11/china-and-south-korea-examining-theresolution-of-the-thaad-impasse/ 
Reuters. (2016, 21 de febrero). U. S. rejected North Korea peace talks offer before last nuclear test: State Department. Reuters. Recuperado de https:// www.reuters.com/article/us-northkorea-nuclear-iduskcn0vu0xe

Singapore Summit: Read the full text of Trump and Kim's joint statement. (12 June 2018). France 24. Recuperado de http://www.france24.com/ en/20180612-singapore-summit-trump-kim-usa-north-korea-read-fulltext-joint-statement-nuclear

Stevenson, C. (2018, 31 de mayo). Lavrov's North Korea visit a reminder to Trump that Russia cannot be ignored. The Independent. Recuperado de https://www.independent.co.uk/voices/trump-north-korea-meetingrussia-lavrov-kim-jong-un-pyongyang-syria-a8378331.html

The U.s.-North Korean Agreed Framework at a Glance. (August 2017). Arms Control Association. Recuperado de https://www.armscontrol.org/factsheets/agreedframework

Yamaguchi, M. (2017, 15 de diciembre). Japan imposes new sanctions on North Korea. USA Today. Recuperado de https://www.usatoday.com/ story/news/world/2017/12/15/japan-imposes-new-sanctions-northkorea/954749001/ 